\title{
Flowable restorative system has a higher retention rate than conventional resin sealant on primary teeth
}

\author{
Is there any difference in retention rate between a conventional resin sealant \\ and a flowable restorative system used as a pit-and-fissure sealant?
}

\begin{abstract}
Corona SAM, Borsatto MC, Garcia L, Ramos RP, Palma-Dibb RG. Randomized, controlled trial comparing the retention of a flowable restorative system with a conventional resin sealant: one-year follow up. Int J Paediatr Dent 2005; 15:44-50
\end{abstract}

Design A split-mouth randomised controlled trial conducted over a 1 -year period.

Intervention A conventional resin-based fissure sealant (Fluroshield $^{\mathbb{R}}$ Dentsply Caulk, Milfor, DE, USA) was applied on randomly assigned upper/lower primary and permanent molars of one side of the mouth. A flowable restorative system (Bond 1 + Flow-It! (Jeneric/ Pentron, Inc., Wallingford, CT, USA) used as a pit-and-fissure sealant was applied to the contra-lateral side. The procedures were carried out under rubber dam and the occlusion was checked with a carbon marker.

Outcome measure Visual inspection was carried out by one examiner at baseline, 6- and 12-month intervals. The retention was assessed following the criteria proposed by Tonn and Ryge: ${ }^{1}$ TR - total retention, $\mathrm{PL}$ - partial loss and TL - total loss.

Results A total of 40 children, aged between 4 and 7 years were recruited, providing a sample size of 160 teeth (80 pairs), comprising 80 primary and 80 permanent molars ( 40 pairs of each tooth type). For both materials, there was no total loss of sealants placed on either the primary or permanent molars over 1 year. From Fluroshield sealants placed on primary teeth, 33 were completely intact after 6 months and 31 after 1 year. From those placed on permanent molars, no loss of material was observed after 6 months, while partial loss was noticed on $5 \%$ of teeth at 1-year recall. For Flow-It! resin applied on primary molars, partial loss of material was observed in only one sealant after 6 months and in two sealants after 1 year. On permanent teeth, 100\% retention rate was observed over a 1 -year follow up. There was a statistically significant difference $(P<0.01)$ between the sealing materials on primary but not permanent teeth, and, overall, Flow-It! sealants presented a higher retention rate at both 6-month and 1-year evaluations. Significant differences $(P<0.01)$ between baseline and the other evaluation periods were also observed.

Conclusions It may be concluded that the flowable restorative system yielded optimal retention on both primary and permanent molars. Its retention rate was significantly higher than that of the conventional pit-and-fissure sealant on primary teeth.

\section{Commentary}

Fissure sealants are recommended to prevent caries of the occlusal surfaces of permanent molars, but most current guidelines do not recommend them for primary teeth. ${ }^{2}$ This paper compares a conventional resin sealant with a flowable restorative system in both primary and permanent teeth. The study used a split-mouth technique in 40 children aged 4-7 years attending Public Health Service clinics. Randomisation methods are not clearly described but each system was used on a pair of primary and permanent molars on one side of the mouth. It is not clear if first or second primary molars were sealed. The main clinical outcome was sealant retention, with loss being recorded as either partial or total. The same clinician placed and assessed the sealants. There were no drop outs at 12 months, but the authors comment that the dropout rate beyond this point prevented a longer follow-up period. This is unfortunate as a longer follow-up period is desirable.

The results show excellent retention for both systems at 12 months, for permanent teeth (two conventional sealants showed partial loss while the flowable restorative system showed $100 \%$ retention). While there were no total sealant losses for either material in primary teeth the flowable system had only two partial losses at 12 months compared to nine for the conventional system. This was a statistically significant difference for retention of sealants in primary teeth in favour of the flowable system $(P<0.01)$. However, the true test of a sealant is its ability to prevent caries not retention per se, this is not reported here. A recent Cochrane review has suggested that the caries prevalence level of both individuals and the population should be taken into account when considering using fissure sealants. ${ }^{1}$ Neither baseline nor end point caries levels are reported for this group of patients so it is not possible to determine if a difference in caries increment exists between the two materials. Indeed it is possible that if the children were caries free at baseline that the sealants may not have been clinically indicated. Further trials, reporting caries prevalence of included subjects are required to determine if flowable restorative systems are superior to conventional sealants over longer periods.

\section{Practice point}

- There is some evidence to suggest that sealants using flowable restorative systems show improved retention rates, particularly for primary teeth.

\section{Barbara Chadwick}

Paediatric Dentistry Unit, Dental School, Cardiff University, Heath Park, Cardiff, UK

1. Tonn EM, Ryge G. Three year clinical evaluation of four sealants in Los Altos, CA. J Dent Res 1982; 61:331 (Abstract 1379).

2. Ahovuo-Saloranta A, Hiiri A, Nordblad A, Worthington H, Mäkelä M. Pit and fissure sealants for preventing dental decay in the permanent teeth of children and adolescents. The Cochrane Database of Systematic Reviews 2004, Issue 3. Art. No.: CD001830. DOI: 10.1002/14651858.CD001830.pub2.

Evidence-Based Dentistry (2005) 6, 89.

doi:10.1038/sj.ebd.6400342 